\title{
Evidence for an oscillating soliton/vortex ring by density engineering of a Bose-Einstein condensate
}

\author{
I. Shomroni, E. Lahoud, S. Levy and J. Steinhauer *
}

When two Bose-Einstein condensates collide with high collisional energy, the celebrated matter-wave interference pattern appears ${ }^{1}$. For lower collisional energies, the repulsive interaction energy becomes significant, and the interference pattern evolves into an array of grey solitons ${ }^{2,3}$. But the lowest collisional energies, producing a single pair of solitons, have not been probed so far. Here, we report on experiments using density engineering on the healing length scale ${ }^{3,4}$ to produce such a pair of solitons. We see evidence that the solitons evolve periodically between vortex rings and solitons. The stable, periodic evolution is in sharp contrast to the behaviour seen in previous experiments ${ }^{5,6}$ in which the solitons decay irreversibly into vortex rings through the so-called snake instability ${ }^{7-13}$. The evolution can be understood in terms of conservation of mass and energy in a narrow condensate.

We consider two condensates separated in space, and with an initial collisional energy $E_{\mathrm{col}}$, which could be in the form of kinetic energy, or potential energy in a magnetic trap. The condensates are then allowed to collide in the $z$ direction. For negligible interactions, the two colliding condensates will interfere like two non-interacting plane waves, forming a standing-wave interference pattern with wavefunction $\psi \propto \cos (k z)$, where the wavelength of the pattern is $\lambda=2 \pi / k$, and $k$ is given by $\hbar^{2} k^{2} / 2 m=E_{\mathrm{col}} / N$, where $m$ is the atomic mass and $N$ is the number of atoms. The density of the pattern is therefore of the form ${ }^{2} n=|\psi|^{2} \propto \cos ^{2}(k z)$. The initial energy $E_{\text {col }}$ supplies the kinetic energy of the standing wave, proportional to $\partial^{2} \psi / \partial z^{2}$, as well as the negligible interaction energy $N \mu / 4$ (see Supplementary Information). However, if $E_{\text {col }}$ is decreased to become comparable to $N \mu / 4$, the form of the fringes will be modified by interactions. It will become energetically favourable to form an array of grey solitons, rather than a cosine-squared pattern ${ }^{2}$. This soliton formation process is relevant for the operation of an atom interferometer ${ }^{14}$. The criterion $E_{\text {col }}<N \mu / 4$ can be written as $\lambda>4 \pi \xi$, where $\xi$ is the healing length, the approximate width of a grey soliton ${ }^{15,16}$. In other words, as $E_{\text {col }}$ decreases, the wavelength of the standing-wave interference pattern increases until the wavelength reaches the size of a soliton. At this point, the fringes will evolve into solitons. A cosine-squared fringe and a soliton are actually very similar in form, as seen in Fig. 1a,b. As $E_{\text {col }}$ decreases further, the number of solitons will decrease, maintaining conservation of energy ${ }^{3}$. The lowest collisional energies will result in two grey solitons travelling in opposite directions. Such a low collisional energy is achieved in our experiment by initially separating the two condensates by a barrier with a width of the order of $\xi$, as shown in Fig. 1c. The energy of this initial configuration relative to the ground state is of the order of $E_{\text {col }}=N \mu(\xi / L)$, where $L$ is the length of the condensate in the $z$ direction. This is comparable to the energy of a grey soliton computed in one dimension, which is of the order of

$$
E_{\text {sol }}=N \mu(\xi / L)\left(1-v^{2} / c^{2}\right)^{3 / 2},
$$

where $v$ is the subsonic soliton velocity and $c$ is the speed of sound in the condensate ${ }^{13} . E_{\mathrm{col}}$ is thus sufficient to create a pair of solitons.

Solitons can also be created by phase engineering ${ }^{17-19}$. There is a phase step $\Delta \phi \leqslant \pi$ across the grey soliton, given by $\Delta \phi=2 \cos ^{-1}(v / c)$. By imposing this phase step, the density profile will evolve into that of a soliton. We can understand this process by considering the current density $\mathbf{J}=(\hbar / m) n \nabla \phi$. By imposing the phase step, this current density is significant only in the region of the step. This corresponds to a divergence in the flow field, $\nabla \cdot \mathbf{J}=\partial J_{z} / \partial z \neq 0$. As the condensate obeys the continuity equation $\nabla \cdot \mathbf{J}=-\partial n / \partial t$, the divergence depletes the density, forming the soliton minimum.

We use density engineering to form solitons, a complementary technique to phase engineering. We impose the density profile, and the phase evolves into that of a soliton. The process is illustrated in Fig. 1d, which shows two solitons separated by a distance $d$. They are moving in opposite directions, with the corresponding opposite phase steps. Our initial configuration shown in Fig. 1c can be thought of as the $d=0$ case of Fig. 1d. Our initial configuration thus corresponds to two overlapping, counterpropagating solitons.

In a nearly one-dimensional (1D) condensate, solitons are stable objects $^{20,21}$. In contrast, in 3D, the stable nonlinear modes are vortex rings, as well as hybrids of solitons and vortex rings ${ }^{22}$. In previous studies in $3 \mathrm{D}$, grey solitons were seen to decay irreversibly into vortex rings through the snake instability ${ }^{7-12}$, which was originally measured in optical solitons ${ }^{13}$. We find a very different, oscillatory behaviour, shown in Fig. 2 by a simulation of the 3D GrossPitaevskii equation ${ }^{15}$ (GPE). The soliton evolves into a vortex ring, which subsequently evolves back into a soliton. This process repeats itself many times with a period of 7-8 ms. We call this a 'periodic soliton/vortex ring', which involves the repeated revival of a soliton, which is an unstable object in our system. The simulation shows that the periodic soliton/vortex ring is stable, surviving the reflection from the end of the condensate, as well as the subsequent collision in the middle of the condensate. The periodic soliton/vortex ring has a constant phase step $(0.7 \pi$ for our experiment), as shown in Fig. $2 \mathrm{j}, \mathrm{k}$.

First, we will explain the evolution of the soliton into a vortex ring in terms of conservation of energy. Owing to the higher density near the centre of the condensate, the soliton moves fastest in this region ${ }^{18}$. This causes the soliton to curve ${ }^{18}$, as shown in Fig. 2f. Owing to the curvature, the area of the soliton has increased, corresponding to an increase in the soliton energy. To 

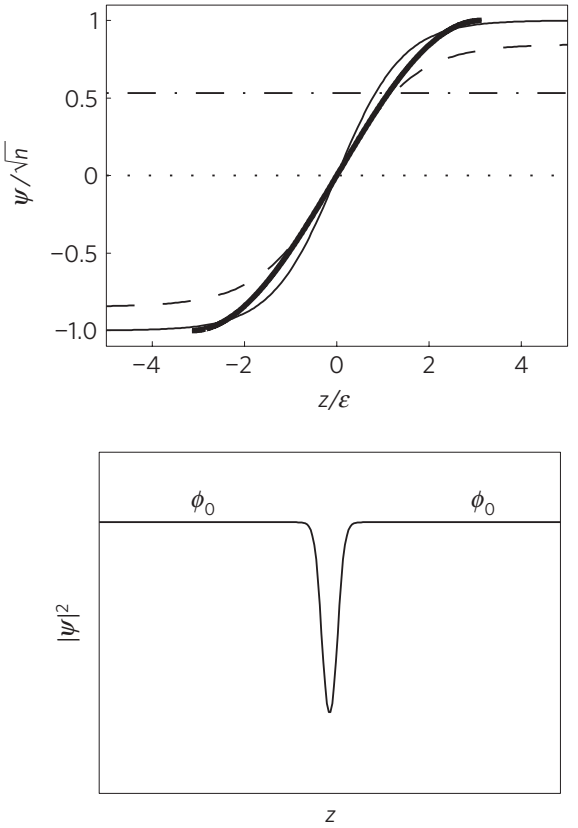

b
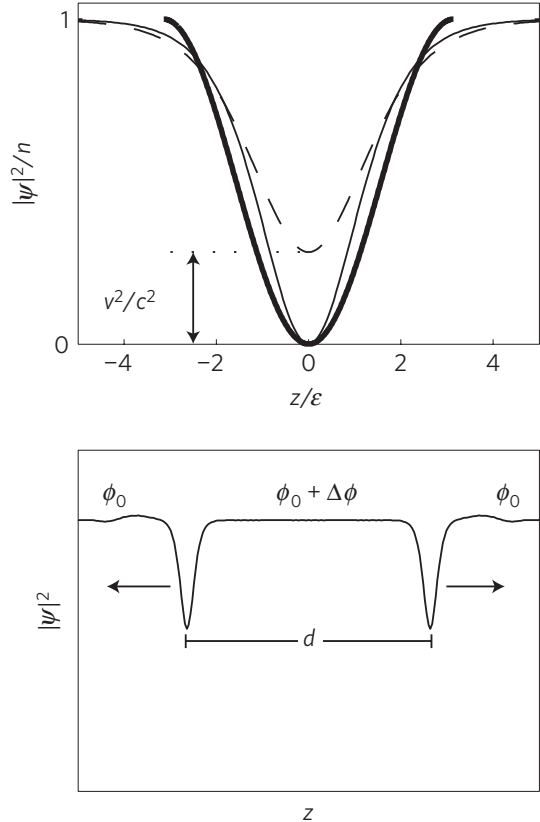

Figure 1 | Solitons and matter-wave interference fringes. a, The order parameter near the transition from interference fringes to solitons. The bold solid curve shows an interference fringe with $\lambda=4 \pi \xi$. The thin solid curve shows a soliton with $v=0$. Both of these order parameters are real. The dashed (dashed-dotted) curve indicates the real (imaginary) part of a grey soliton, with $v=0.53 \mathrm{c}$. $\mathbf{b}$. The density associated with the curves of part $\mathbf{a}$. $\mathbf{c}$, The initial density and phase of our experiment, created by density engineering. $\mathbf{d}$, The density and phase of two counterpropagating solitons.

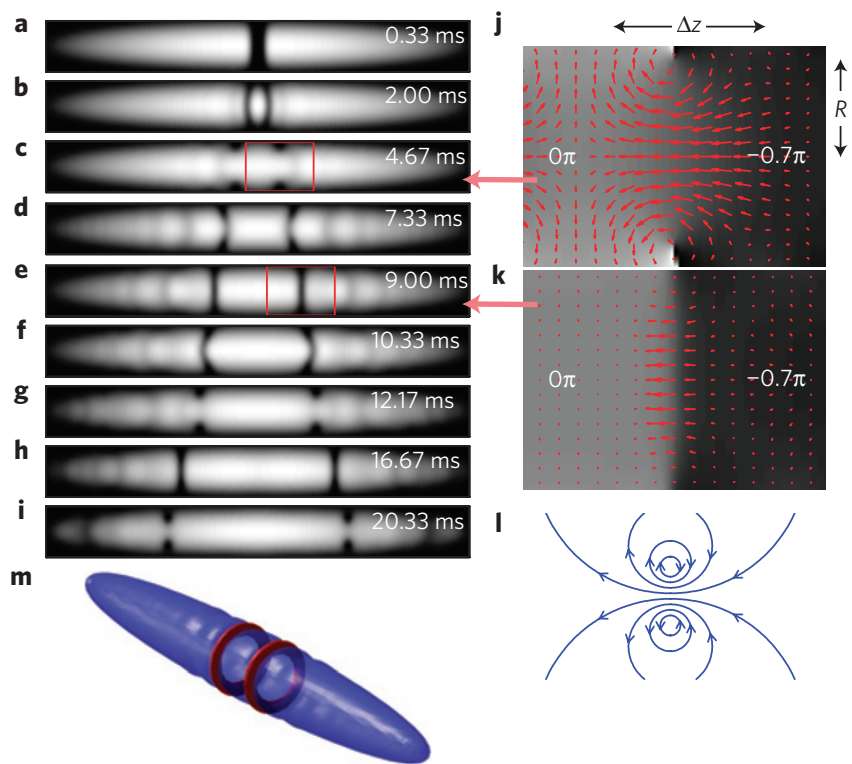

Figure 2 | Simulation of the periodic soliton/vortex ring. This simulation shows $3 \times 10^{4}$ atoms. $\mathbf{a}-\mathbf{i}$, Slices of the condensate. The shades of grey indicate the density. $\mathbf{a}$, The condensate during the turn-off of the barrier. $\mathbf{b}, \mathbf{e}, \mathbf{h}$, The soliton stage of the periodic soliton/vortex ring. $\mathbf{c}, \mathbf{g}, \mathbf{i}$, The vortex ring stage. The axis of the two counterpropagating vortex rings is horizontal in the image. The slice of the vortex ring appears as two dark spots, separated vertically. $\mathbf{d}, \mathbf{f}$, The transitions between the soliton and vortex ring stages. $\mathbf{j}$, The shades of grey indicate the phase, and the red vectors indicate $\mathbf{J}$. The image is an enlargement of the area between the red lines in c. The values 0 and $-0.7 \pi$ indicate the phase on either side of the phase step. $\mathbf{k}$, As in $\mathbf{j}$, except that the enlargement refers to $\mathbf{e}$. I, An illustration of the slice of a vortex ring in an infinite, homogeneous condensate. $\mathbf{m}, \mathrm{An}$ illustration of the two counterpropagating vortex rings of $\mathbf{c}$, showing a surface of constant density (blue) as well as a surface of constant flow speed (red) conserve the energy of the soliton, the increase in area must be compensated by an increase in $v$, by equation (1). This corresponds to a reduction in the depth of the soliton, as shown in Fig. 1b. The soliton thus moves with ever-increasing speed and decreasing depth, until the density minimum vanishes altogether, leaving a vortex ring, as shown in Fig. $2 \mathrm{c}$,g,i. The appearance of the vortex ring does not violate Kelvin's theorem (conservation of circulation), because the vortex core can enter and exit the condensate through the nearby boundary ${ }^{23}$.

The fate of vortex rings in a quantum fluid is a long-standing field of study ${ }^{23,24}$. In superfluid ${ }^{4} \mathrm{He}$ at non-zero temperatures, it was suggested that the radius of a vortex ring shrinks in time owing to dissipation, until it evolves into a roton. We can understand the very different evolution of our vortex ring into a soliton in terms of conservation of mass. We consider a vortex ring in an infinite homogeneous condensate ${ }^{15,25}$, as illustrated ${ }^{24}$ in Fig. 2l. The density is constant in time, maintained by the zero divergence of the flow $(\nabla \cdot \mathbf{J}=0)$. In other words, after the flow traverses the centre of the vortex ring from right to left, it returns to the right outside the vortex ring. In contrast, our vortex ring is close to the edge of the condensate, as shown in Fig. 2c,g,i. The flow lines therefore have no return path to the right side, causing $\nabla \cdot \mathbf{J} \neq 0$. The divergence depletes the density, forming the soliton minimum. This situation is very similar to that of phase engineering of a condensate described above. In fact, the vortex ring has a phase step, as shown in Fig. 2 j. The phase step occurs on a length scale $\Delta z$ of the order of the radius of the vortex ring, which is also the radius $R$ of the condensate. Thus, for creating solitons from vortex rings, $R$ should not be too much larger than $\xi$. This can also be understood energetically. The periodic soliton/vortex ring requires that the soliton and the vortex ring have the same energy. The ratio of these energies contains a factor of $R / \xi$, placing an upper limit on $R$. Using the GPE simulation, we find that the vortex ring evolves into a soliton for $R \leqslant 13 \xi$, corresponding to $N \leqslant 7 \times 10^{4}$ for our trap frequencies. Note that it is the radial variation of the condensate rather than the axial variation that causes the evolution. 


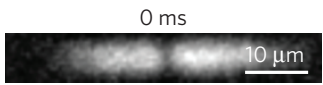

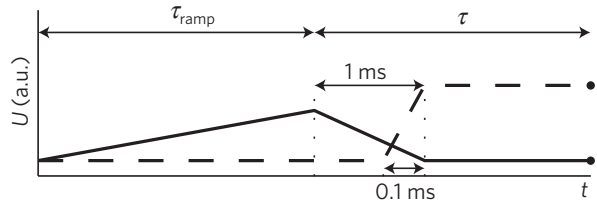
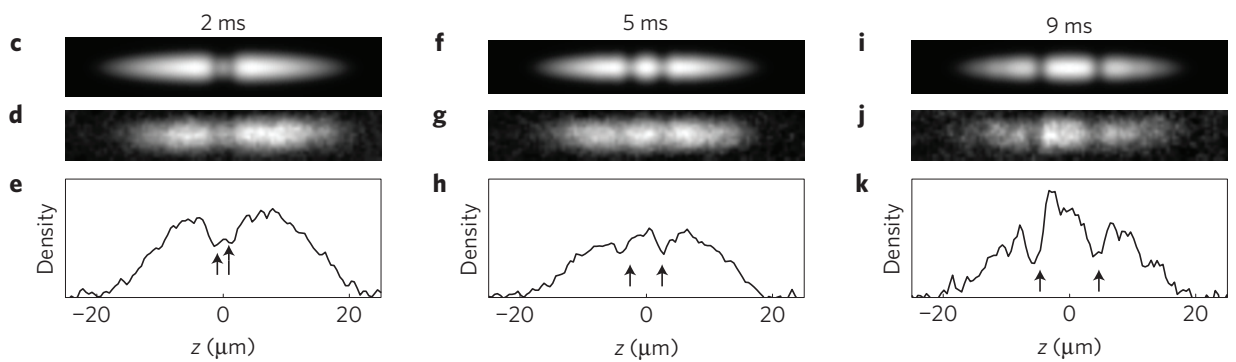

Figure 3 | In situ images of the vortex rings and solitons. The experimental parts of the figure show averages of 2-6 destructive phase contrast images, using small detuning. The simulations show integrated images including finite resolution and depth of field. The condensates contain $1 \times 10^{4}$ atoms $(R=6 \xi)$. $\mathbf{a}$, The initial condition with the barrier on. $\mathbf{b}$, The height of the barrier as a function of time (not to scale). The solid curve corresponds to density engineering of solitons. The dashed curve corresponds to the creation of sound pulses. $\mathbf{c}$, Simulation of the initially formed pair of solitons at 2 ms. $\mathbf{d}$, In situ image of the two counterpropagating solitons at $2 \mathrm{~ms}$. e, Profile of $\mathbf{d}$, along the central axis of the image. The arrows indicate the positions of the solitons from the simulation in c. f, Simulation of the vortex ring stage at $5 \mathrm{~ms}$. $\mathbf{g}$, In situ image of two counterpropagating vortex rings at $5 \mathrm{~ms}$. $\mathbf{h}$, Profile of $\mathbf{g}$, along the central axis. The arrows indicate the positions of the vortex rings from the simulation in $\mathbf{f}$. $\mathbf{i}$, Simulation of the soliton stage at $9 \mathrm{~ms}$. $\mathbf{j}$, In situ image of two counterpropagating solitons at $9 \mathrm{~ms}$. $\mathbf{k}$, Profile of $\mathbf{j}$, along the central axis. The arrows indicate the positions of the solitons from the simulation in $\mathbf{i}$.

We carry out density engineering through a narrow, blue-detuned laser barrier, which is adiabatically ramped up in $\tau_{\text {ramp }}=200 \mathrm{~ms}$ to a height $U=h(3,000 \mathrm{~Hz})$, creating a Bose-Einstein condensate (BEC) with a density minimum, as shown in Fig. 3a. The potential is then rapidly turned off in $1 \mathrm{~ms}$, resulting in the initial condition illustrated in Fig. 1c. This sequence is indicated by the solid curve in Fig. 3b. As seen in Fig. $3 \mathrm{~d}$, the two counterpropagating solitons can be distinguished after $2 \mathrm{~ms}$, which result from the interference between the two condensates in Fig. 3a, colliding with low energy. Figure $3 \mathrm{~d}$ corresponds to Fig. 1d. Figure $3 \mathrm{c}$ shows the simulation at $2 \mathrm{~ms}$ with imaging effects, including integration of the density perpendicular to the image, finite resolution and finite depth of field. The experimental images in Fig. 3 agree well with the simulated images. The resolution is taken to be $2 \mu \mathrm{m}$, which gives the best visual agreement between the experiment and the simulation. This is larger than the measured $1.2 \mu \mathrm{m}$ resolution of the imaging system. We attribute this difference to defocusing of the imaging system with its short depth of field.

After a time $\tau=5 \mathrm{~ms}$, we obtain in situ images of the pair of counterpropagating vortex rings of the periodic soliton/vortex ring, as shown in Fig. 3g. In the image, the two vortex rings are seen as weak density minima. The weak density minima are also seen in Fig. 3h, which shows the profile along the central axis in Fig. 3g. Owing to the shape of a vortex ring, the density minima should be weak along this central axis. After each of the two vortex rings has evolved back into a soliton, we image the soliton stage at $\tau=9 \mathrm{~ms}$, as shown in Fig. 3j. Two strong, slit-like solitons are seen. Figure 3k shows the greatly increased contrast along the central axis in the soliton stage, relative to the vortex ring stage in Fig. $3 \mathrm{~h}$. This increase in contrast is evidence for the evolution of the vortex ring into a soliton. We find that the solitons are more visible for the small atom number shown in the figure.

To measure the speed of the periodic soliton/vortex ring, we observe its position as a function of time, as shown in the integrated profiles in Fig. $4 \mathrm{a}$, and the filled circles in Fig. $4 \mathrm{~b}$. We see that the speed (the slope) is approximately constant in time, and is therefore the same for the soliton stage and the vortex ring stage. The speed is observed to be $v=0.66 \pm 0.08 \mathrm{~mm} \mathrm{~s}^{-1}$, for $N=3 \times 10^{4}$. This speed is of the same order of magnitude as the speed $v \approx(\hbar / 2 m R) \ln (1.59 R / \xi) \approx 0.3 \mathrm{~mm} \mathrm{~s}^{-1}$ predicted for a vortex ring in an infinite, homogeneous condensate ${ }^{15,23,24}$. Excellent agreement between experiment and simulation is seen throughout Fig. 4.

The speed of a grey soliton is less than $c$, so it is interesting to compare the speed of the periodic soliton/vortex ring with $c$. We measure $c$ using the technique of ref. 26. Specifically, we rapidly turn on the barrier in $100 \mu$ s and leave it on as indicated by the dashed curve in Fig. 3b, creating two counterpropagating sound pulses, as shown after $5 \mathrm{~ms}$ in Fig. $4 \mathrm{~g}$. The profiles in Fig. $4 \mathrm{c}$ and the open circles in Fig. $4 \mathrm{~b}$ show the position of the sound pulses as a function of time, giving $c=1.24 \pm 0.07 \mathrm{~mm} \mathrm{~s}^{-1}$, which agrees with the theoretical value ${ }^{27}$ of $c=\sqrt{\mu / 2 m}=1.15 \pm 0.09 \mathrm{~mm} \mathrm{~s}^{-1}$. We thus find that the periodic soliton/vortex ring moves slower than the speed of sound, at $v=0.53 c$. This subsonic motion is evident in Fig. 4e,g, in that the two arrows are closer together for the vortex rings than for the sound pulses.

In conclusion, we have achieved density engineering of a pair of counterpropagating grey solitons, which are the result of a low-energy collision between two BECs. The close relationship between solitons and matter-wave interference fringes illustrates the quantum mechanical nature of solitons in a BEC. Owing to the inhomogeneous nature of the narrow condensate, each soliton evolves into a periodic soliton/vortex ring. As vortex rings and solitons can be considered to be quasiparticles ${ }^{8,15,22,23}$, perhaps the periodic soliton/vortex ring could also be described as a Rabi oscillation between these quasiparticle states. Such a Rabi oscillation is seen in optics, between two soliton states ${ }^{28}$.

After submission of our manuscript, we learned of ref. 29, reporting density engineering of pairs of solitons. As the condensate was nearly one-dimensional, the solitons were stable.

\section{Methods}

Our experimental apparatus is basically described in ref. 30. Potentials are focused onto the condensate through an aspheric lens with numerical aperture 0.5 . Imaging is carried out through this same lens. After reaching the BEC phase, the trap frequencies are adiabatically reduced by half in $350 \mathrm{~ms}$ to 117 and $13 \mathrm{~Hz}$ in the radial and axial directions, respectively. The resulting condensate contains $1 \times 10^{4}$ or $3 \times 10^{4}{ }^{87} \mathrm{Rb}$ atoms, as specified in the captions of Figs 3 and 4 . The condensate with $3 \times 10^{4}$ atoms has $\mu / h=580 \mathrm{~Hz}$ ( $h$ is Planck's constant), corresponding to a minimum healing length of $\xi=0.32 \mu \mathrm{m}$, which is found at 


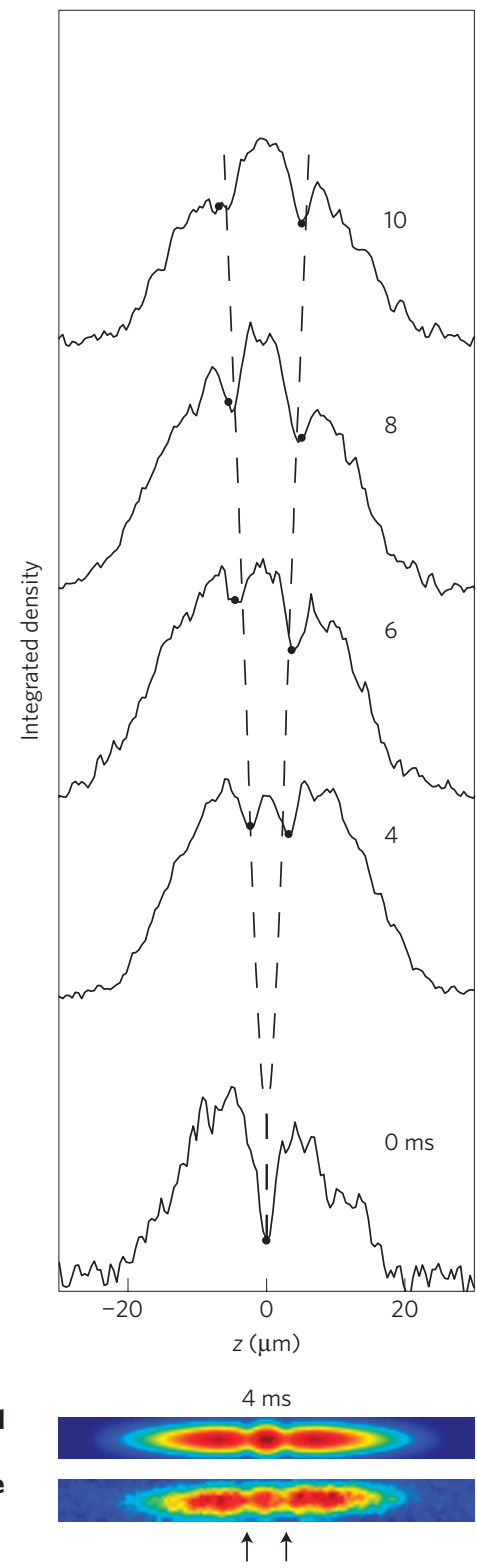

b

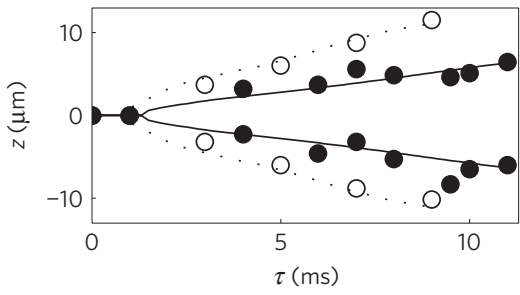

c

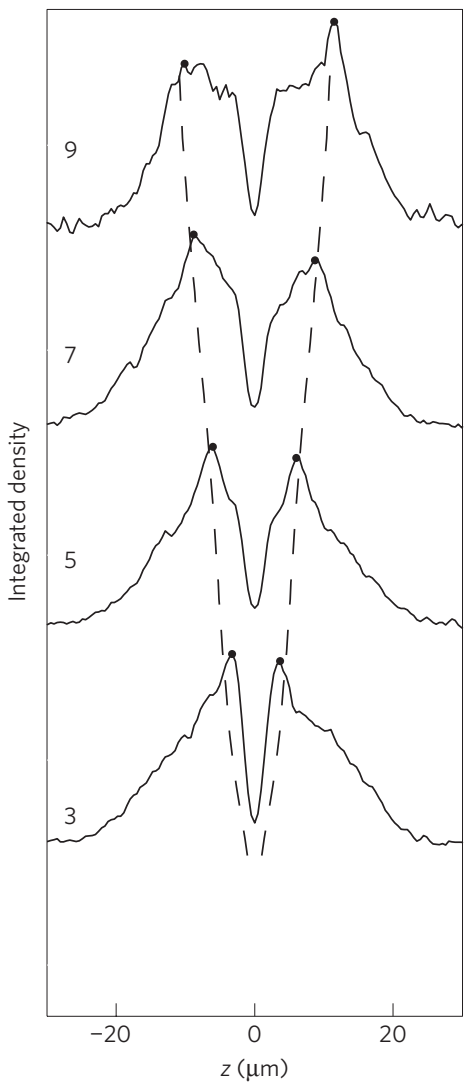

$\mathbf{f}$

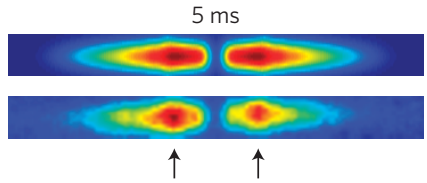

Figure 4 | The speed of the periodic soliton/vortex ring. The condensates contain $3 \times 10^{4}$ atoms $(R=10 \xi)$. Each profile and image is an average of several images, except for the $\tau=0$ ms profile. $\mathbf{a}$, Integrated profiles of the periodic soliton/vortex ring. The dashed curves are the result of the simulation. $\mathbf{b}$, Filled circles indicate the position of the periodic soliton/vortex ring as a function of time. Open circles indicate the position of the sound pulses. The solid and dotted curves are simulations of the periodic soliton/vortex ring and sound pulses, respectively. c, The sound pulses. The dashed curves indicate the simulation. d, Simulation of the vortex ring stage at $4 \mathrm{~ms}$. e, In situ image of two counterpropagating vortex rings at $4 \mathrm{~ms}$. The arrows indicate the positions of the vortex rings from the simulation in d. f, Simulation of counterpropagating sound pulses at $5 \mathrm{~ms}$. $\mathbf{g}$, In situ image of two counterpropagating sound pulses at $5 \mathrm{~ms}$. The arrows indicate the positions of the sound pulses from the simulation in $\mathbf{f}$.

the centre of the condensate. The healing length is larger at the lower densities found away from the centre.

The laser barrier is blue-detuned by $6 \mathrm{~nm}$ from the $780 \mathrm{~nm}$ resonance. The condensate is split in the radial direction by this highly elongated laser beam with an axial diameter of $1.4 \mu \mathrm{m}$, which is $4.4 \xi$ (3.4 $\xi$ ) for the larger (smaller) condensate. Using the 1D GPE simulations of ref. 3, as well as our 3D GPE simulations, this diameter of roughly $4 \xi$ is the maximum that will create a single pair of solitons.

\section{Received 14 April 2008; accepted 5 December 2008;} published online 18 January 2009

\section{References}

1. Andrews, M. R. et al. Observation of interference between two Bose condensates. Science 275, 637-641 (1997).
2. Scott, T. F., Ballagh, R. J. \& Burnett, K. Formation of fundamental structures in Bose-Einstein condensates. J. Phys. B 31, L329-L335 (1998).

3. Carr, L. D., Brand, J., Burger, S. \& Sanpera, A. Dark-soliton creation in Bose-Einstein condensates. Phys. Rev. A 63, 051601(R) (2001).

4. Reinhardt, W. P. \& Clark, C. W. Soliton dynamics in the collisions of Bose-Einstein condensates: An analogue of the Josephson effect. J. Phys. B 30, L785-L789 (1997).

5. Anderson, B. P. et al. Watching dark solitons decay into vortex rings in a Bose-Einstein condensate. Phys. Rev. Lett. 86, 2926-2929 (2001).

6. Dutton, Z., Budde, M., Slowe, C. \& Hau, L. V. Observation of quantum shock waves created with ultra-compressed slow light pulses in a Bose-Einstein condensate. Science 293, 663-668 (2001).

7. Kadomtsev, B. B. \& Petviashvili, V. I. On the stability of solitary waves in weakly dispersing media. Sov. Phys. Dokl. 15, 539-541 (1970). 
8. Jones, C. A., Putterman, S. J. \& Roberts, P. H. Motions in a Bose condensate: V. Stability of solitary wave solutions of non-linear Schrödinger equations in two and three dimensions. J. Phys. A 19, 2991-3011 (1986).

9. Josserand, C. \& Pomeau, Y. Generation of vortices in a model of superfluid ${ }^{4} \mathrm{He}$ by the Kadomtsev-Petviashvili instability. Europhys. Lett. 30, 43-48 (1995).

10. Feder, D. L., Pindzola, M. S., Collins, L. A., Schneider, B. I. \& Clark, C. W. Dark-soliton states of Bose-Einstein condensates in anisotropic traps. Phys. Rev. A 62, 053606 (2000).

11. Brand, J. \& Reinhardt, W. P. Solitonic vortices and the fundamental modes of the 'snake instability': Possibility of observation in the gaseous Bose-Einstein condensate. Phys. Rev. A 65, 043612 (2002).

12. Theocharis, G., Frantzeskakis, D. J., Kevrekidis, P. G., Malomed, B. A. \& Kivshar, Y. S. Ring dark solitons and vortex necklaces in Bose-Einstein condensates. Phys. Rev. Lett. 90, 120403 (2003).

13. Mamaev, A. V., Saffman, M. \& Zozulya, A. A. Propagation of dark stripe beams in nonlinear media: Snake instability and creation of optical vortices. Phys. Rev. Lett. 76, 2262-2265 (1996).

14. Jo, G.-B. et al. Phase-sensitive recombination of two Bose-Einstein condensates on an atom chip. Phys. Rev. Lett. 98, 180401 (2007).

15. Pitaevskii, L. \& Stringari, S. Bose-Einstein condensation Sects 5.4 and 5.5 (Oxford Univ. Press, 2003).

16. Jackson, A. D., Kavoulakis, G. M. \& Pethick, C. J. Solitary waves in clouds of Bose-Einstein condensed atoms. Phys. Rev. A 58, 2417-2422 (1998).

17. Burger, S. et al. Dark solitons in Bose-Einstein condensates. Phys. Rev. Lett. 83, 5198-5201 (1999).

18. Denschlag, J. et al. Generating solitons by phase engineering of a Bose-Einstein condensate. Science 287, 97-101 (2000).

19. Becker, C. et al. Oscillations and interactions of dark and dark-bright solitons in Bose-Einstein condensates. Nature Phys. 4, 496-501 (2008).

20. Muryshev, A. E., van Linden van den Heuvell, H. B. \& Shlyapnikov, G. V. Stability of standing matter waves in a trap. Phys. Rev. A 60 , R2665-R2668 (1999).
21. Carr, L. D., Clark, C. W. \& Reinhardt, W. P. Stationary solutions of the one-dimensional nonlinear Schrödinger equation. I. Case of repulsive nonlinearity. Phys. Rev. A 62, 063610 (2000).

22. Komineas, S. \& Papanicolaou, N. Nonlinear waves in a cylindrical Bose-Einstein condensate. Phys. Rev. A 67, 023615 (2003).

23. Donnelly, R. J. Quantized Vortices in Helium II Chs 1, 4 (Cambridge Univ. Press, 1991).

24. Rayfield, G. W. \& Reif, F. Quantized vortex rings in superfluid helium. Phys. Rev. 136, A1194-A1208 (1964).

25. Guilleumas, M., Jezek, D. M., Mayol, R., Pi, M. \& Barranco, M. Generating vortex rings in Bose-Einstein condensates in the line-source approximation. Phys. Rev. A 65, 053609 (2002).

26. Andrews, M. R. et al. Propagation of sound in a Bose-Einstein condensate. Phys. Rev. Lett. 79, 553-556 (1997).

27. Zaremba, E. Sound propagation in a cylindrical Bose-condensed gas. Phys. Rev. A 57, 518-521 (1998).

28. Snyder, A. W., Hewlett, S. J. \& Mitchell, D. J. Periodic solitons in optics. Phys. Rev. E 51, 6297-6300 (1995).

29. Weller, A. et al. Experimental observation of oscillating and interacting matter wave dark solitons. Phys. Rev. Lett. 101, 130401 (2008).

30. Levy, S., Lahoud, E., Shomroni, I. \& Steinhauer, J. The a.c. and d.c. Josephson effects in a Bose-Einstein condensate. Nature 449, 579-583 (2007).

\section{Acknowledgements}

We thank A. Soffer, M. Segev, W. Ketterle, A. Minguzzi and R. Ozeri for helpful discussions. This work was supported by the Israel Science Foundation.

\section{Additional information}

Supplementary Information accompanies this paper on www.nature.com/naturephysics. Reprints and permissions information is available online at http://npg.nature.com/ reprintsandpermissions. Correspondence and requests for materials should be addressed to J.S. 